\title{
A High-Performance Ka-band Cryo-Cooled Receiver for Deep Space Applications
}

\author{
A. Mediavilla ${ }^{\# 1}$, J.L. Cano ${ }^{\# 2}$, D. Vegas ${ }^{\#}$, E. Artal ${ }^{\#}$, A. Tazon ${ }^{\#}$, L.M. de la Fuente ${ }^{\#}$, C. Chambon", B. Fauroux ${ }^{*}$, \\ R. Rayet ${ }^{*}$, S. Rawson", S. Halté \\ \#DICOM, University of Cantabria, Santander, Spain \\ ${ }^{*}$ CALLISTO FRANCE, Villefranche du Lauragais, France \\ ${ }^{\wedge}$ ESA-ESOC, Darmstadt, Germany \\ \{1angel.mediavilla, 2juanluis.cano\}@unican.es
}

\begin{abstract}
This paper describes the design of a ground station antenna feed system, that includes feed horn, polarizer, rejection filter, mono-pulse tracking coupler and low noise amplifiers with polarisation switching. The entire feed system assembly is cooled to cryogenic temperature $(T<10 \mathrm{~K})$ in order to maximise the $G / T$ of the ground station antenna. The cryogenic cooling system described is fully redundant and this novel design allows one cryocooler to be removed and replaced, while the other cryocooler is still running. The design has been submitted for patent protection.
\end{abstract}

Keywords - ground stations, antenna feed, cryogenic electronics, space exploration, deep space antenna.

\section{INTRODUCTION}

ESA interplanetary missions are more and more demanding in terms of scientific data return and the 35-m ESA deep-space antennas will require significant G/T improvement in order to close the communication link with remote space probes.

Although current ESA deep-space antennas include cryocooled Low Noise Amplifiers (LNA) to improve sensitivity without increasing antenna gain, the overall noise performance of the complete receiver is mainly limited by the loss of the feed components which are operated at room temperature. As it is very difficult to lower insertion loss of passive components and develop LNAs with significantly lower noise temperature $\left(T_{N}\right)$, the most efficient strategy is to cool down as many of the feeder components as possible to cryogenic temperature. An additional improvement is obtained by implementing a $\mathrm{TE}_{21}$ mono-pulse tracking coupler in the RF chain to compensate any antenna pointing error which could significantly degrade the antenna gain at $\mathrm{Ka}$ band. The overall objective of the work described in this paper was: (1) to design a cryogenic feeder including antenna horn, tracking coupler, polarizer, filters and cryogenic LNAs and (2) to manufacture and test a prototype. The prototype has now undergone acceptance tests. In the future the prototype will be upgraded to become an operational unit. ESA will deploy a Ka-band cryo-cooled feed in 2020 to support BepiColombo and JUICE missions with an expected downlink data rate improvement of $80 \%$.

This new generation of receiver will replace the current room temperature feeder system and three cryogenic LNAs (two operational and one spare units). Several functionalities were required by ESA to meet operational constraints. Firstly, on the RF side, a proper redundancy concept based on an electro-mechanical cryogenic transfer switch has been implemented to redirect the received signal to the redundant channel in case of LNA failure. Secondly, the feed components are cooled down to around $T=9 \mathrm{~K}$ physical temperature using two cryogenic coolers (nominal mode). If one cooler is defective, an innovative technical solution has been developed to be able to disconnect and replace a faulty cryogenic cooler while the second one is still operated. In this case (degraded mode), the physical temperature on the cryo cooled RF chain is around $T=11.5 \mathrm{~K}$. Doing this, the best trade-off has been achieved between maintaining high performance of the receiver operated at cryogenic temperature in the case of a failure of a key components, whilst avoiding outage time for the operation.

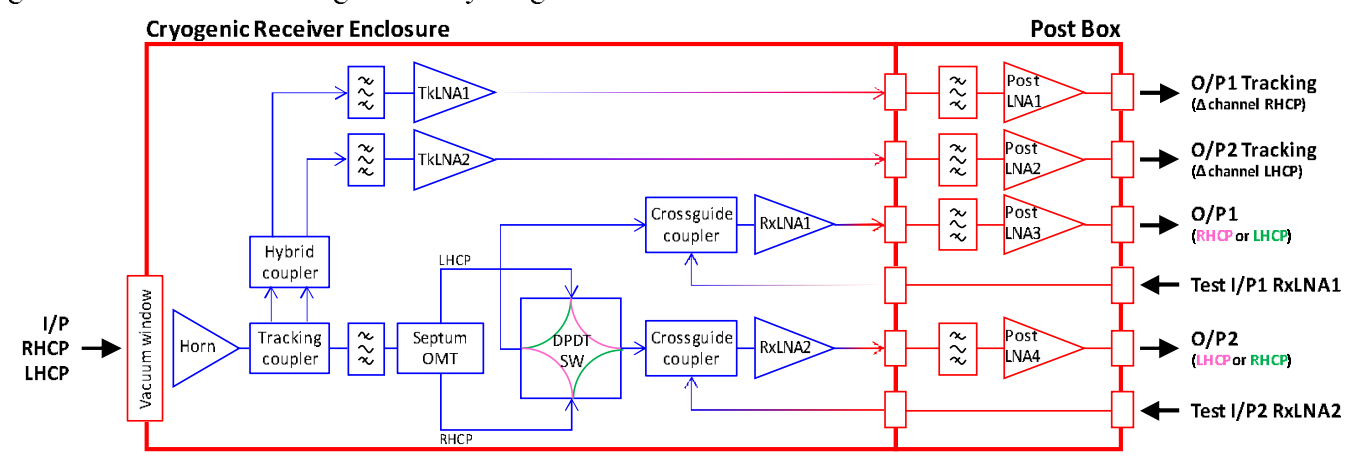

Fig. 1. Schematic diagram of the Ka-band cryogenic feed. 


\section{RECEIVER SYSTEM DESCRIPTION}

The Ka-band functionality of the ESA deep space antennas is to receive circularly polarized signals in the $31.8-32.3 \mathrm{GHz}$ frequency range while maintaining a high degree of rejection at 30,34 and $40 \mathrm{GHz}$ with an axial ratio better than $0.5 \mathrm{~dB}$ within $1 \mathrm{~dB}$ contour. Furthermore, the feed gain at the LNA inputs must be greater than $28 \mathrm{~dB}$ while the noise temperature at the horn aperture is being limited to $T_{N}=25 \mathrm{~K}$.

For this purpose, all the critical components of the designed cryogenic system are enclosed in a dedicated Dewar. In Fig. 1, the red parts are room temperature components whereas blue parts are RF components operated at cryogenic temperature. The Dewar input interface is a window that shall isolate vacuum established inside the Dewar and be as transparent as possible at Ka-band frequencies. In addition, it shall avoid water condensation on the window layer and withstand the force due to the pressure difference. The design is based on pressure and vacuum windows made of Mylar with another expanded polystyrene foam layer [1] used to maintain and support mechanically the vacuum window which is put under severe stress. The RF chain includes a feed horn, the $\mathrm{TE}_{21}$ tracking coupler, the low loss circular filter, the septum polarizer and the electro mechanical cryo switch. Several cryo-LNA are implemented to obtain a very sensitive receiver. Other standard elements such as rectangular waveguide sections, bends or injection couplers are less relevant in terms of noise addition. Room temperature post-amplifiers and filters are included to fulfil system requirements in terms of gain, out of band rejection and output compression point. Fig. 2 shows a crosssection sketch of the Dewar with all the front-end components assembled.

\section{A. Feed Horn}

This element represents a real challenge because at $32 \mathrm{GHz}$ a conical corrugated horn having $29 \mathrm{~dB}$ of gain results in 500 $\mathrm{mm}$ long, and cannot be assembled in this kind of receiver. To overcome this drawback a dual shaped (sinusoidal + exponential) corrugated horn was designed to reduce the axial length by $30 \%$ while maintaining the specified gain and aperture diameter. The throat section was carefully designed to guarantee a $\mathrm{TE}_{11}$ return loss better than $35 \mathrm{~dB}$, a crosspolarization level better than $40 \mathrm{~dB}$ and sidelobes below $36 \mathrm{~dB}$. Moreover, the horn return loss for the $\mathrm{TE}_{21}$ modes is around 24 $\mathrm{dB}$.

\section{B. $T_{21}$ Tracking Coupler}

Conventional $\mathrm{TE}_{21}$ travelling couplers represent an elegant and broadband solution that needs at least $200 \mathrm{~mm}$ in length at Ka-band [2]. To minimize again the axial length of the feed, a resonant four-slot $\mathrm{TE}_{21}$ mode coupler in oversized circular waveguide was designed for this application where the final overall length is around $40 \mathrm{~mm}$. By cascading a $90^{\circ}$ hybrid coupler at the tracking ports, RHCP or LHCP error signals can be independently extracted and routed towards a conventional tracking receiver. This compact solution gives a return loss of
$24 \mathrm{~dB}$ at the tracking ports while maintaining a port to port isolation better than $45 \mathrm{~dB}$. This will assure a null depth of around $40 \mathrm{~dB}$ in the delta radiation pattern, which is largely enough for tracking purposes.

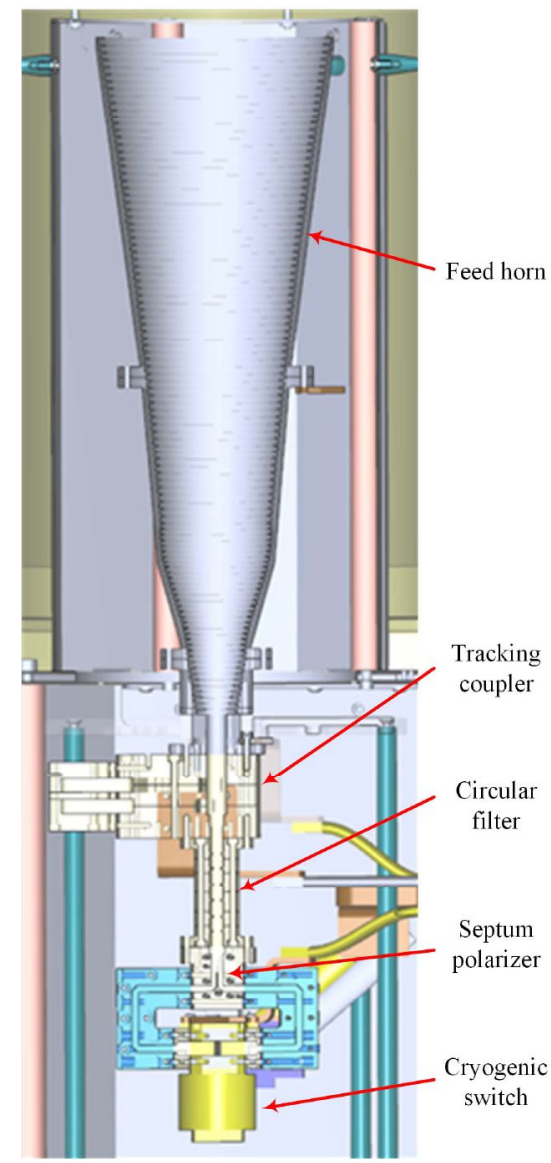

Fig. 2. Cross-section sketch of the developed receiver in the Dewar.

\section{Circular Common Filter}

In most of the existing cryo receivers the pre-LNA filtering operation is done in rectangular waveguide, that is, one filter per receiving channel. This means a large amount of mass to be cooled down. The proposed architecture uses a single circular iris coupled filter located in between the tracking coupler and the septum polarizer. As the filter was made of aluminium with a further silver plating, the design process took into account the silver thickness as well as the dimensional variation when cooled. The frequency shift from room temperature to $T=15 \mathrm{~K}$ cryogenic temperature was around $100 \mathrm{MHz}$ upward and the insertion loss decreased down to $0.08 \mathrm{~dB}$.

\section{Septum Polarizer}

This conventional circuit separates the two RHCP/LHCP incoming signals into two separate rectangular ports. As the key point is losses, the component was designed to be fabricated in two halves by using a special milling technique that seals the junction thus minimizing the mating losses. The input/output return loss is better than $30 \mathrm{~dB}$ while the measured insertion 
loss remains in the $0.04 \mathrm{~dB}$ range. It should be pointed out here that this element was re-optimized along with the tracking coupler in order to be ensure that the overall axial ratio remains below $0.5 \mathrm{~dB}$.

\section{E. Electromechanical Cryo Switch}

The cryogenic switch is essential to provide the RF redundancy function. By actuating the switch at cryogenic temperature, it is possible to steer either RHCP or LHCP signals in one or the other channel. Thus, if one channel is defective, the other channel is still able to receive signals. To achieve this, a commercial waveguide switch has been specifically modified to be able to operate reliably at cryogenic temperature. Because measuring the insertion loss of the switch at cryogenic temperature is very difficult, a test bench has been designed to measure the noise temperature of a receiver with and without the cryo switch assembled in the chain. Between both configurations, the noise temperature was measured $0.1-0.2 \mathrm{~K}$ higher with the switch. Insertion loss of the switch has been found extremely low and estimated at around $0.01-0.02 \mathrm{~dB}$ at a physical temperature of $T=25 \mathrm{~K}$. Moreover, the switch has been actuated more than 10,000 times at cryogenic temperature and no defects were observed. The noise temperature was measured with a very good repeatability before and after the long-term test, which proved the high-performance and the reliability of the switch at cryogenic temperature.

\section{F. Cryo LNAs}

Two types of high-performance cryogenic LNAs have been evaluated during the project. A commercial cryogenic LNA (reference LNF-LNC23_43WA) has been purchased form Low Noise Factory (LNF, Gothenburg, Sweden). Two prototypes of cryogenic LNAs have been developed by Centro Astronomico de Yebes (CAY, Yebes, Spain) with new InAs-HEMT from ETHZ (Zurich, Switzerland). Table 1 summarizes the RF performance measured on both amplifiers.

Table 1. Cryogenic LNA RF performance summary.

\begin{tabular}{|l|l|l|}
\hline \multirow{2}{*}{ Parameters at 15K } & \multicolumn{2}{|c|}{ Specified value over 31.8-32.3GHz } \\
\cline { 2 - 3 } & LNC23 43WA & CAY YKa series \\
\hline Frequency range & $23-42 \mathrm{GHz}$ & $25.5-32.3 \mathrm{GHz}$ \\
\hline Noise temperature & $7 \mathrm{~K}$ max & $10 \mathrm{~K}$ max \\
\hline Gain & $27.5 \mathrm{~dB}$ mean & $33.5 \mathrm{~dB}$ mean \\
\hline Return losses & $10 \mathrm{~dB}$ min input & $12 \mathrm{~dB}$ min input \\
& $14 \mathrm{~dB}$ min output & $15 \mathrm{~dB}$ min output \\
\hline Power consumption & $10 \mathrm{~mW}$ & $18 \mathrm{~mW}$ \\
\hline PoutldB & $-10 \mathrm{dBm}$ typical & $-5 \mathrm{dBm}$ typical \\
\hline
\end{tabular}

\section{SYSTEM MEASUREMENT RESULTS}

After completing the preliminary and detailed design phases, the RF chain has been manufactured and tested. Radiation patterns with antenna feed horn and tracking coupler have been also verified for the fundamental and the tracking modes. As it can be seen at Fig. 3, the $\mathrm{TE}_{11}$ mode gain is $29.2 \mathrm{~dB}$ in boresight direction at $32.05 \mathrm{GHz}$, associated with a cross polarization of $37 \mathrm{~dB}$ within $1 \mathrm{~dB}$ contour. This leads to an axial ratio of 0.13 $\mathrm{dB}$. For the $\mathrm{TE}_{21}$ tracking mode, the null depth is around $39 \mathrm{~dB}$ at $32.05 \mathrm{GHz}$ and tracking slope is around $0.1 \mathrm{~V} / \mathrm{V} / \mathrm{deg}$ up to 4 deg theta angle as shown in Fig. 4.

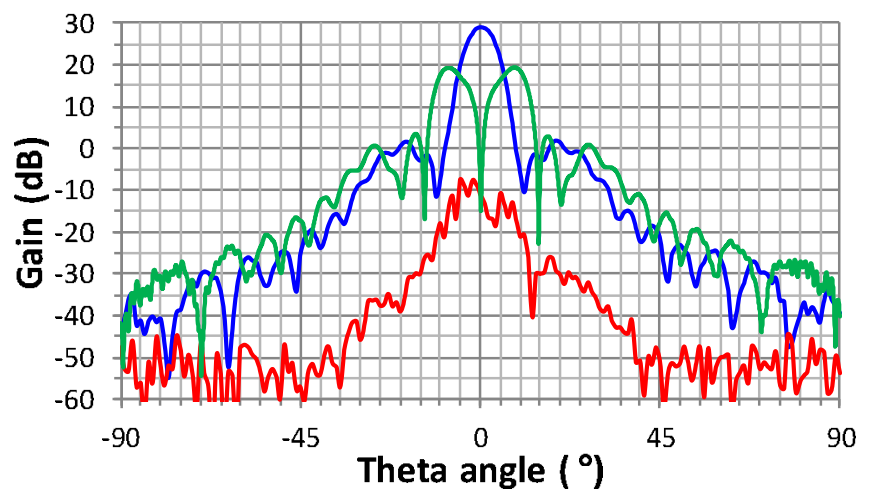

Fig. 3. $\mathrm{TE}_{11}$ and $\mathrm{TE}_{21}$ radiation patterns measured at $32.05 \mathrm{GHz}$ and for $0 \mathrm{deg}$ cut plane. Co-polar (blue line) and cross-polar (red line) of mode $\mathrm{TE}_{11}$. $\mathrm{TE}_{21}$ radiation pattern is plotted with a green line.

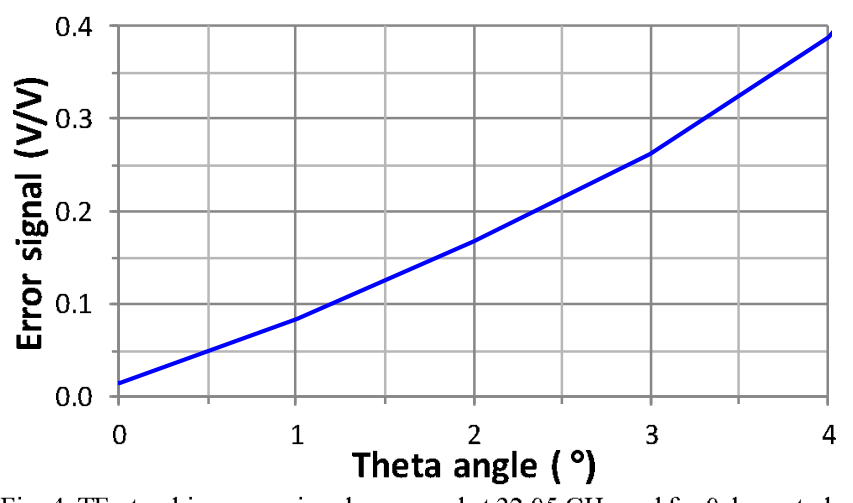

Fig. 4. $\mathrm{TE}_{21}$ tracking error signal measured at $32.05 \mathrm{GHz}$ and for 0 deg cut plane.

After RF chain validation, a Ka-band cryo cooled feed prototype Dewar has been successfully manufactured and assembled at Callisto laboratory. A picture of the whole system is presented in Fig. 5. The overall dimensions are around $610 \mathrm{x}$ $1560 \mathrm{~mm}$ (including a cold head extraction tool) and its weight is in the order of $200 \mathrm{~kg}$.

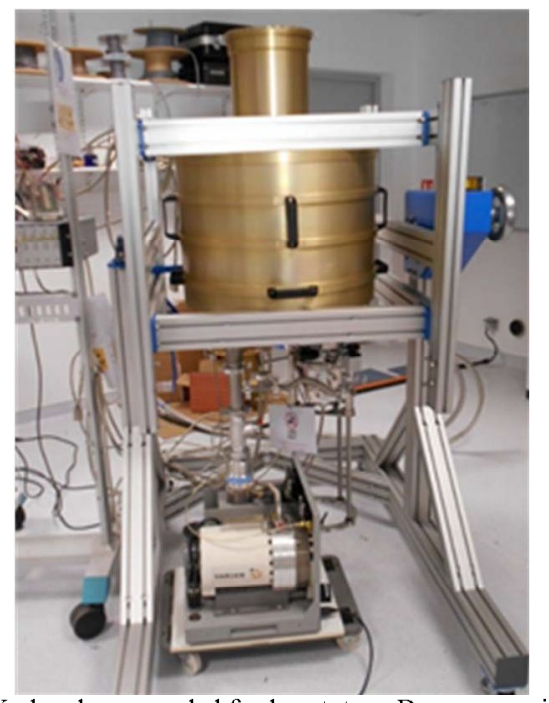

Fig. 5. Ka-band cryo cooled feed prototype Dewar overview. 
As the complete RF chain with feed is cryogenically cooled inside a single enclosure, the cryocooler system becomes a single point of failure. It was then required to have redundant cryocoolers running on the receiver. However, when one of the two cryocoolers requires regular maintenance or even fails, the $\mathrm{RF}$ chains must remain at cryogenic temperatures allowing noise temperature to be low enough so as not to stop the ground station operations. To allow for replacement of the failed cooler while keeping the second cooler running, a custom sleeve system (called "double sleeve") has been designed and successfully tested. It is basically a sealed inner sleeve with a second outer sleeve encapsulating the first one. The outer sleeve is attached to the cold head and connected via a set of clamps to the inner sleeve. By means of the clamps, the inner sleeve can be thermally uncoupled from the outer one and the cold head can be dismounted without any risk while the outer sleeve is still at cold temperature. The double sleeve is equipped with a hydraulic system to apply high linear force to extract the double sleeve at cryogenic temperature and under vacuum. The following Fig. 6 shows the temperature profile inside the receiver at various points whilst one double sleeve and cold head is extracted and the second cooler keeps running and maintaining the RF components at cryogenic temperature. After a successful reconnection of the extracted cooler and consecutive cooldown, the nominal cryogenic temperatures are recovered $(\mathrm{T}=9 \mathrm{~K})$.

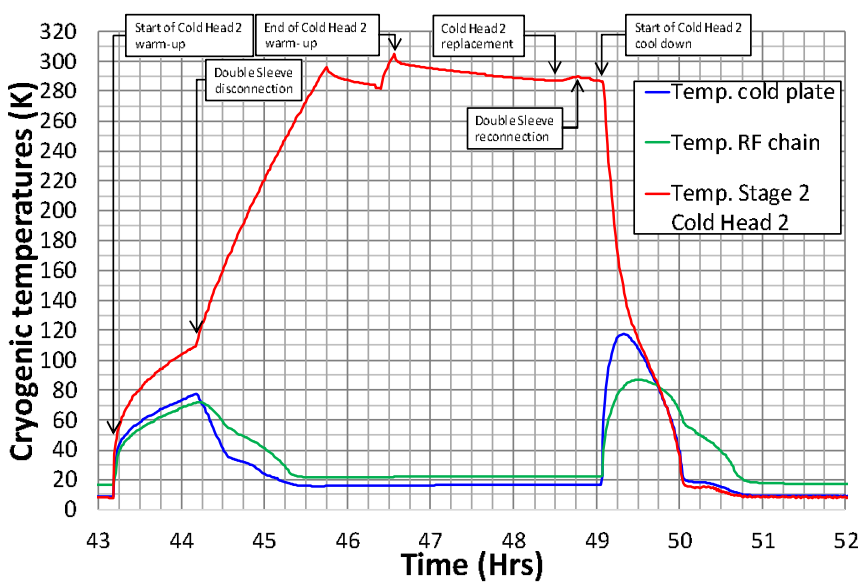

Fig. 6. Double sleeve extraction and temperature recovery under cryogenic operations.

Noise temperature has been tested using two RF matched loads with very well-known noise temperatures. The warm one is a room temperature absorbent foam set in front of the input aperture of the feed horn, the second is a cold load when antenna feed horn points towards clear sky. To calculate the cold sky noise, the sky brightness temperature is integrated within the antenna radiation patterns for $\mathrm{TE}_{11}$ and $\mathrm{TE}_{21}$ modes [3]. Noise temperature has been measured in both RHCP/LHCP channels and for both positions of the redundancy switch. The maximal noise temperature is around $T_{N}=17 \mathrm{~K}$ over $31.8-32.3 \mathrm{GHz}$ as presented in Fig. 7.

Gain and group delay have been measured by using cross guide cryogenic couplers ahead LNAs and test inputs with around $31 \mathrm{~dB}$ of coupling factor. For the configuration with commercial cryogenic LNA, the gain is $58.9 \pm 0.5 \mathrm{~dB}$ and the group delay maximal variation is $0.5 \mathrm{~ns}$ over $31.8-32.3 \mathrm{GHz}$ and $0.06 \mathrm{~ns}$ per $20 \mathrm{MHz}$. The rejection is within specification and is found around $140 \mathrm{dBc}$ at $30 \mathrm{GHz}, 120 \mathrm{dBc}$ at $34 \mathrm{GHz}$ and $125 \mathrm{dBc}$ at $40 \mathrm{GHz}$. Finally output compression point is measured at $+12 \mathrm{dBm}$.

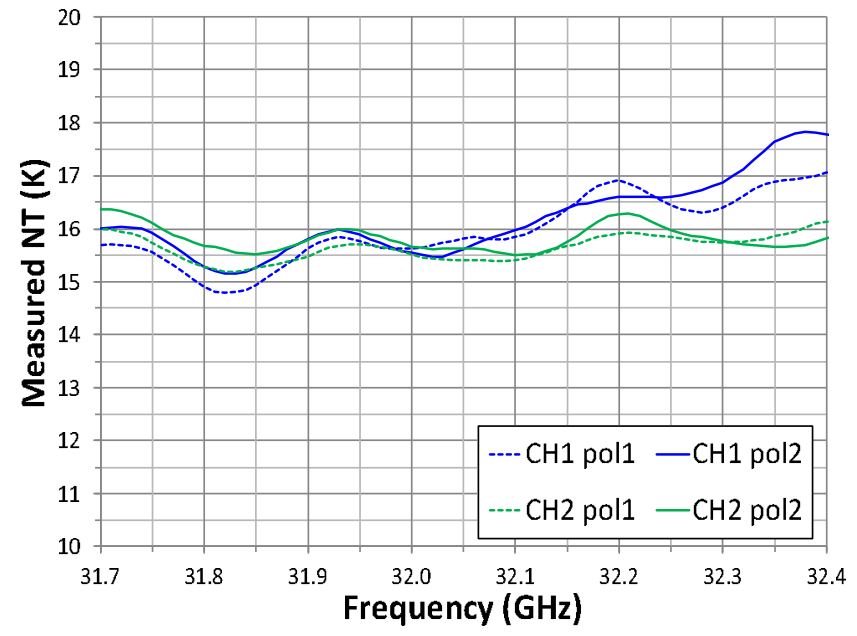

Fig. 7. Noise temperature measured on Ka-band cryo cooled feed prototype Dewar.

\section{CONCLUSION}

A high performance cryo-cooled receiver operating in Kaband has been developed for deep space applications. The proposed receiver will replace the existing systems in the ESA ground stations for deep space communications with improved $\mathrm{RF}$ and cryogenic performance for higher scientific data rates. Moreover, the maintainability has been increased by implementing an innovative redundancy solution for the cryogenic system. Doing this, the receiver can be operated at cryogenic temperature while maintenance is carried out, which reduces outage time during operation.

\section{ACKNOWLEDGMENT}

Ka-Band cryocooled feed project developed under ESA Technology Research Program contract number 4000113406/15/D/JR.

\section{REFERENCES}

[1] Y. Lee, B. Ellison, P. Huggard, M. Harman, A. Boughriet, W. Bartynowski, M. Oldfield, N. Morris, P. Hekman and G. Han Tan, "ALMA Front-End Verification Using a Dry Cold Load" $21^{s t}$ International Symposium on Space Terahertz Technology, Oxford, 2325 March 2010.

[2] L. Sakr, "The higher order modes in the feeds of the satellite monopulse tracking antennas," IEEE Mediterranean Electrotechnical Conf. (MELECON), Cairo, Egypt, May 2002.

[3] T. Y. Otoshi, Noise Temperature Theory and Applications for Deep Space Communications Antenna Systems, Artech House, 2008. 